\title{
LOW COST WIRELESS SENSOR NETWORKS AND SMARTPHONE APPLICATIONS FOR DISASTER MANAGEMENT AND IMPROVING QUALITY OF LIFE
}

\author{
Raghu Teja Mulukutla ${ }^{1}$, Anurag Mondal ${ }^{2}$, Sourabh Bollapragada ${ }^{3}$, Gopal Mulukutla ${ }^{4}$, \\ Sandeep Kumar Ladi $^{5}$ \\ ${ }^{I}$ Student, Department of Electronics and Communication Engineering, GITAM University, Visakhapatnam, AP, \\ India \\ ${ }^{2}$ Student, Department of Electronics and Electrical Engineering, GITAM University, Visakhapatnam, AP, India \\ ${ }^{3}$ Student, Department of Computer Science Engineering, GITAM University, Visakhapatnam, AP, India \\ ${ }^{4}$ Research Scientist, Earth Systems Research Center, Institute for the Study of Earth, Oceans and Space, University of New \\ Hampshire, Durham, NH, US \\ ${ }^{5}$ Assistant professor, GITAM University, Visakhapatnam, AP,India
}

\begin{abstract}
Spatially distributed Wireless Sensor Networks (WSN) comprising low-cost components (sensors, Microcontrollers, wireless communication devices) provide a way to measure several quantitative variables of nature and the human environment (air, temperature, soil temperature, precipitation and water quality). Owing to the increased human intervention in nature, there is a rise in unpredictability of weather and natural environment. This causes human induced disasters such as long dry periods, flash floods, subsequent landslides, heat-island effect, sea-level rise, beach erosion. Very often the effects of disasters natural or manmade are regional and early warning infrastructure may not be sufficient. In this paper we address disaster preparedness and prevention by utilizing low cost WSNs to monitor the region and designingfreeAndroid cloud application to instantly notify users, an occurrence of a disaster. The deployment of the WSN is done using various interconnected nodes across the region. Each node consists of a low cost Arduino Microcontroller, Xbee radio communication module and sensors which measure data substantial to detect a disaster. Each node collects data and sends through line of sight to a central station. This station is a server which collects and sends the data to the cloud where it is stored and inspected for detecting a dangerous situation. The cloud also maintains the sensor data in an online database periodically. We will demonstrate a smartphone application which communicates with the cloud so that notifications are sent in real time when a disaster is detected to alert the users located within the WSN area, helping in early evacuation, finding nearby safety shelters etc. We show that by establishing a synergy between Smartphone and reliable modern sensors like ground motion sensors(for early warning of earthquakes), soil moisture sensors (for early warning of drought), flow sensors (for early warning offloods) in WSN will improve the quality of life by ensuring safety against disasters.
\end{abstract}

Keywords_disaster prevention, wireless network, smartphone alerts during disaster, early evacuation, safety shelters, early detection, disaster alert, sensors, disaster, flow sensor, ground motion sensor.

\section{INTRODUCTION}

Although many developments have been made in disaster prediction and risk reduction, there is a lot of ground left to catch up. Early warning systems, which are generally designed to cater to a wider regions many a times fail to be equally effective in notifying region specific hazardous events. Recent increase of human intervention in nature is causing disruptions in the local weather patterns and also to an extent climate. These disruptions make it increasingly tough for making accurate predictions in local weather and environmental occurrences. As early warning systems depend on these predictions, their efficacy is seriously challenged.
With more emphasis given on setting up national warning centers rather than local centers, there is a compromise being made on detecting potentially hazardous scenarios which are specific to a region. Clearly in some cases of emergencies, a robust localized approach would generate a faster response to the situation. For example, local detection of forest fires will ensure a quick remedy action before it escalates to a potential national disaster. Moreover, events due to human error, such as gas leakages, collapsing buildings, fire accidents and dam flooding are detectable easier when the approach is local.

One of the major setbacks in establishing a local-warning system is the cost. A community based early warning system might not be a priority task for 
economically backward rural areas. However, owing to the rapid advancements in the realms of electronics and software in the present, modern digital world, usage of open source electronics and software counterparts, the cost of establishing a localized warning system can be reduced by a great extent.
This paper discusses a low-cost model of a regional warning system comprising a Wireless Sensor Network (WSN) and a Smartphone application. This model is an interdisciplinary approach involving four abstraction levels:

- Sensor Network

- Gateway

- Cloud

- Android Application

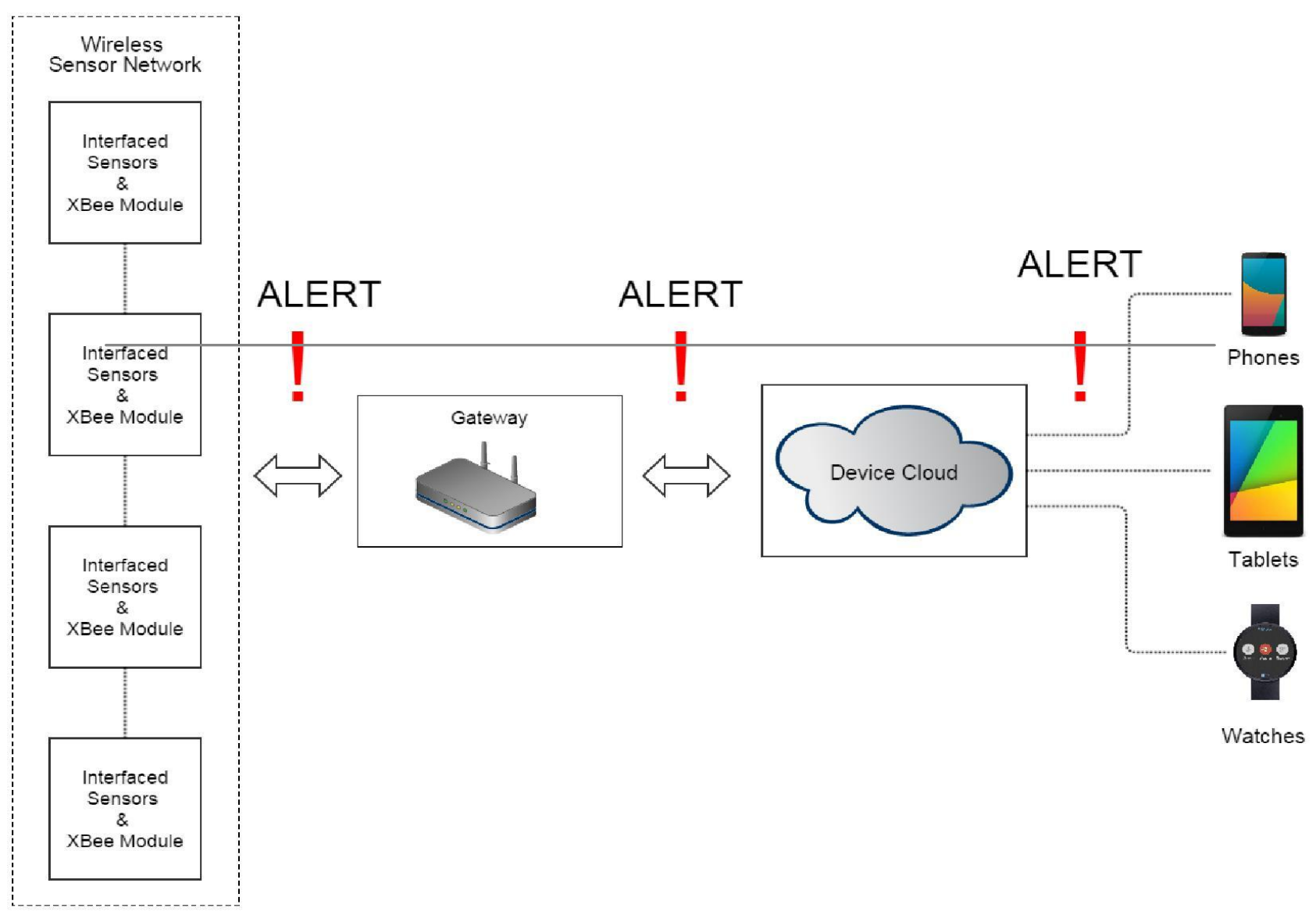

Fig 1 Model Overview

\section{SENSOR NETWORK}

The Sensor network is deployed over a region to be monitored. The network consists of nodes constantly working in tandem, sensing threats by coordinating the gathered sensor data measured from observable environmental parameters2.

Each node consists of sensors such as temperature, geophone, flood, humidity, $\mathrm{pH}$ glass electrodes and many more, interfaced with a microcontroller. A ZigBee3 based radio frequency transmitter/receiver module is used to form a network.

\subsection{Microcontroller System}

Our model uses an open-source Arduino Mega 2560 microcontoller board, which is based on ATmega2560, an 8-bit Atmel microcontroller. Sensors can be easily interfaced and monitored by programming the microcontroller board in $\mathrm{C}$ language.

\subsection{RF Communication}

For the purpose of communicating the sensor data, $\mathrm{XBee}$ Pro, a low power radio module is interfaced with the microcontroller system.

The XBees modules communicate between each other in the network using ZigBee protocol[1] which is based on IEEE Standard 802.15.4. Each XBee assumes one of these three roles -coordinator, router and end point for a streamlined communication network. However for any network it is necessary for having one coordinator. 


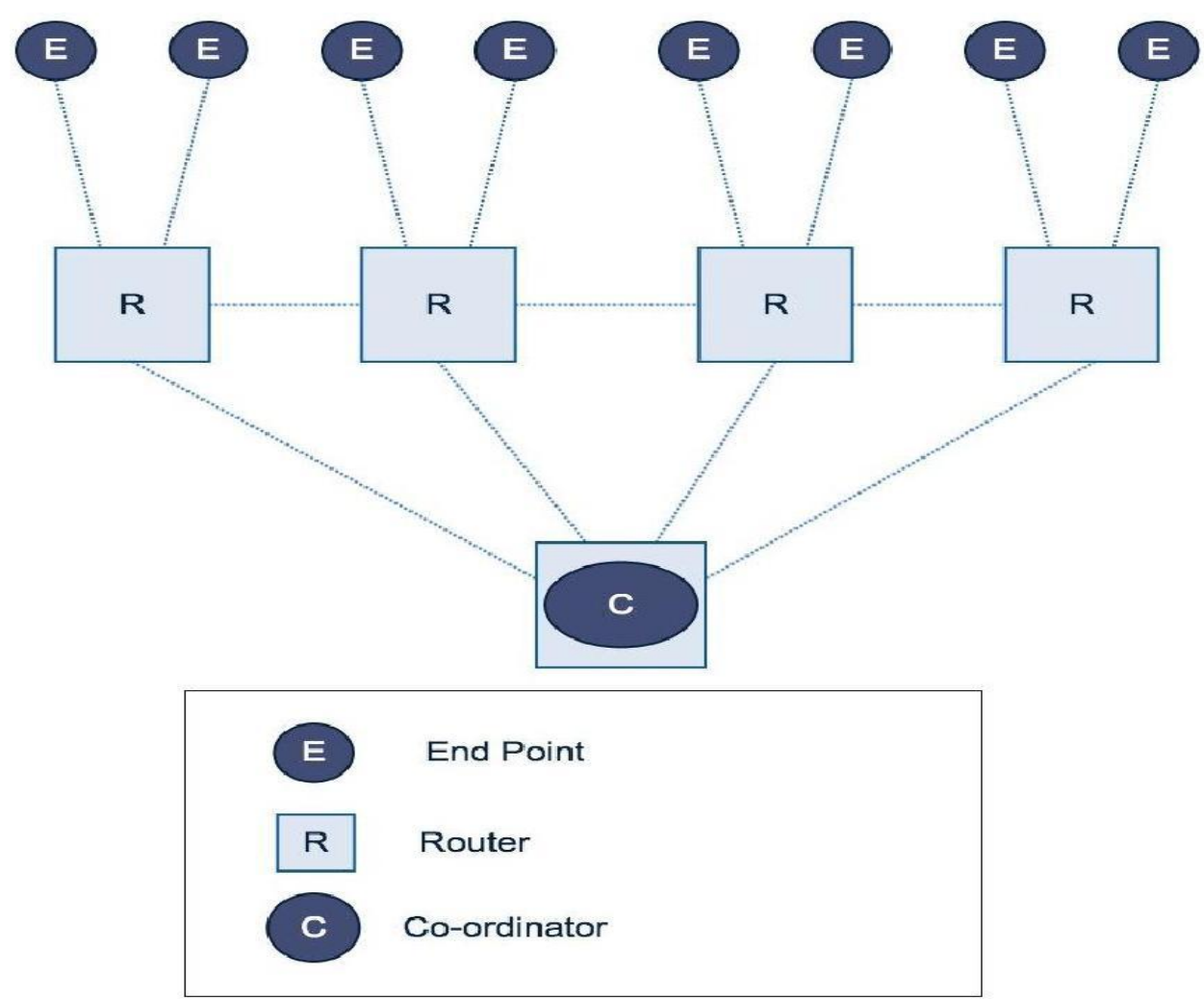

Fig 2 Mesh Configuration of XBees in Network

\subsection{Sensors}

Measurable physical quantities such as temperature, humidity, toxicity of gases, ground vibrations and alike are crucial in detecting a threat. A simple example would be interfacing a commercially available temperature sensor with the microcontroller system, for analysis of spikes in temperature. These spikes are a precursor to a possible fire breakout in the environment being monitored. The microcontroller system can be programmed to trigger a signal, in real-time, relaying it across the abstraction levels, to the smartphone, in case the temperature gathered from the sensor spikes up above a set threshold. The availability of low-cost, high-grade and easily interfaceable sensors allow us to measure a number of physical quantities and sense variety of threats.

\begin{tabular}{|l|l|l|}
\hline $\begin{array}{l}\text { S.N } \\
\text { o. }\end{array}$ & Sensor name & Related Function \\
\hline 1 & $\begin{array}{l}\text { Temperature/Hu } \\
\text { midity }\end{array}$ & $\begin{array}{l}\text { Detecting fires(Forest Fires, } \\
\text { Building Fires) }\end{array}$ \\
\hline 2 & Geophone & $\begin{array}{l}\text { Detecting ground vibrations } \\
\text { (Earthquake) }\end{array}$ \\
\hline 3 & Water Alarm & $\begin{array}{l}\text { Detecting flooding(Dam, } \\
\text { riverbed) }\end{array}$ \\
\hline 4 & Weather Shield & $\begin{array}{l}\text { Locally detecting inclement } \\
\text { weather }\end{array}$ \\
\hline 5 & Gas Breakout & $\begin{array}{l}\text { Detecting } \\
\text { leakages(LPG, CO) }\end{array}$ \\
\hline
\end{tabular}

Fig 3 Table showing how varied sensors can be used in detecting a disaster.

\section{GATEWAY}

The Gateway is a networking device which is used to connect the Sensor Network to the Internet. It does this by converting the packet format received from the sensor network into the TCP/IP format which is used to send data on the Internet. It has cellular, Wi-Fi and Ethernet WAN options for flexible broadband connectivity.

The Xbee network connects to the Digi Gateway and feeds the sensor data over the Internet to the cloud. Python scripts can be run on the gateway for extending functionalities. Additionally, the Gateway gathers the GPS location of each nodes present in the network, which is then used to inform the location of the occurrences to the users of the application.

\section{CLOUD}

A Cloud is an aggregation of several computers which allow centralized storage and online access to resources and services.

The Digi Gateway connects to Etherios Device Cloud over the Internet. Device Cloud allows the remote configuration, monitoring, management and data access of each XBee present in the sensor network.

In a promising approach, various Data Mining4 techniques employed on the sensor data play a primary role in, statistical analysis and predicting future occurrences using data trend analysis. 


\section{ANDROID APPLICATION}

The sensor data directed to the Device Cloud forms a platform for developing smartphone applications which function as real-time notification providing an easily accessible warning system5.The application uses the Etherios Device Connector APIs for connecting with the Device Cloud. This allows the mobile application to communicate with the Cloud and alert the users in case of emergency.

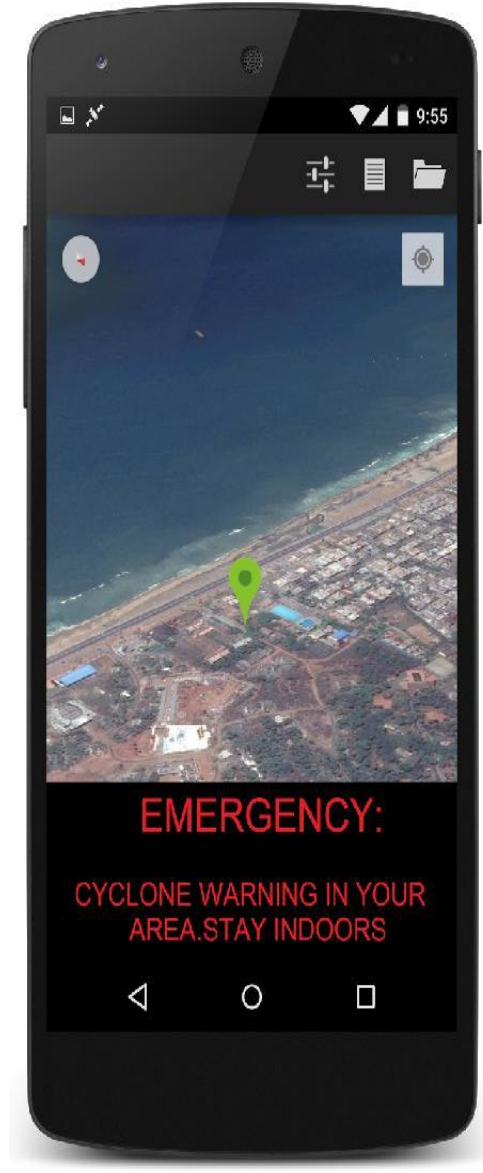

(A)

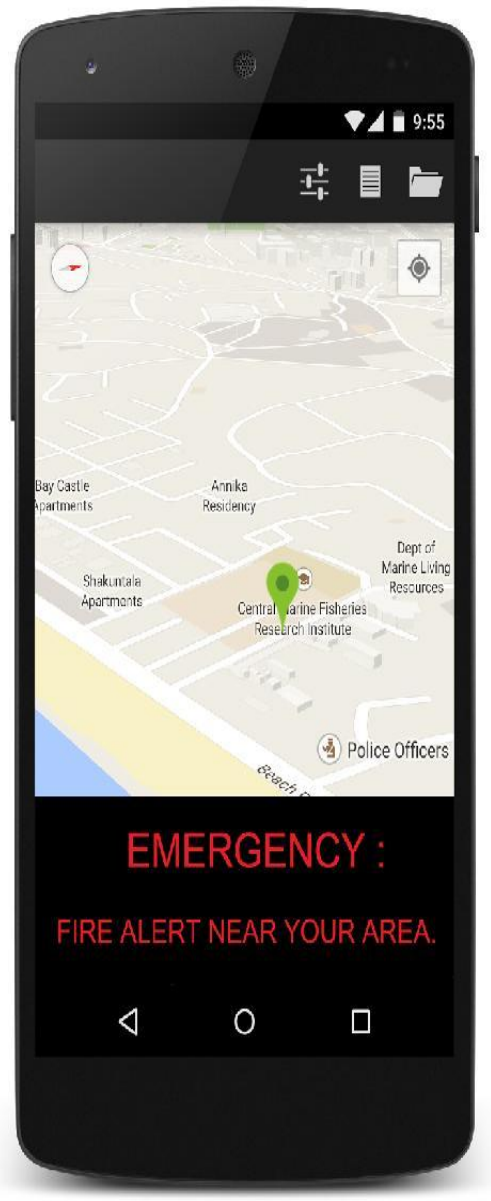

(B)
Along with alerts, a GIS UI provided in the application, shows insightful information about the disaster situation. The Map is also useful in routing the users to safety shelters, thereby expediting relief operations. As long as connectivity persists, smartphones can help accelerate the promulgation of disaster alert messages over a wider range of users.

Fig 4 Screenshots of Android applications running on a phone. Figure 4(A) \& (B) Show an automated emergency notification. Figure4(C) shows navigation services to route users to safety zones.

\section{RELIABILITY CONCERNS}

A drawback of this model may be that the electronic components present in the WSN are susceptible to physical damage during disaster occurrences resulting in a disconnection. To combat such nodal disconnections, the WSN's networking protocol is designed to be "self-healing", re-routing communication paths automatically despite the disconnection of few member nodes. This means that the WSN will continue to function even after few of the nodes stop operating.

Another concern of this model would be on powering the nodes present in the WSNs, especially when the area to be monitored is remote and does not have access to power lines. As operating voltages are in compliance, the nodes can be powered by solar cells during the daytime and with solar powered battery banks during nightfall.

\section{COST \& SCALABILITY CONCERNS}

The extensive usage of open source technologies and low-cost high-grade sensor components in this model reduces the economic burden and allows for high customizability at any stage. Moreover, almost non existent licensing helps local communities to adapt the model quicker without glitches. The WSN supports easy scalability. Nodes can be easily added to an already set-up WSN by simple configuration. 


\section{CONCLUSION}

The interdisciplinary and customizable nature of this model opens up new ways in which we can improvise disaster management systems, improving their efficacy.The WSNs in conjunction with smartphone technology will establish a highly effective regional early warning system.

The goal of this model is to detect hazardous situations real-time and send alert messages almost instantaneously, leaving enough time to initiate emergency evacuation or similar operations. This ensures a feeling of safety in a community, which plays a primal role in improving quality of life.

\section{REFERENCES}

[1]. Sensor Networks for Disaster Relief Operations Management by Erdal Cayirci and Tolga Coplu.

[2]. Environmental Wireless Sensor Networks By Peter Corke, Tim Wark, Raja Jurdak, Wen Hu.

[3]. ZigBee: A Low Power Wireless Technology For Industrial Applications by Nishak and Yask.

[4]. Data Mining: Concepts and Techniques by Jiawei Han and Micheline Kamber.

[5]. Implementation of an Android-Based Disaster Management System by Jovilyn Therese. 